\title{
Pairwise Coding to Mitigate Polarization Dependent Loss
}

\author{
Chen Zhu ${ }^{1}$, Binhuang Song ${ }^{1}$, Leimeng Zhuang ${ }^{1}$, Bill Corcoran ${ }^{1,2}$, and Arthur J. Lowery ${ }^{1,2}$ \\ ${ }^{1}$ Electro-Photonics Laboratory, Electrical and Computer Systems Engineering, Monash University, Australia \\ ${ }^{2}$ Centre for Ultrahigh-bandwidth Devices for Optical Systems (CUDOS), Australia \\ Email: arthur.lowery@monash.edu
}

\begin{abstract}
By pre-coding data across polarizations, the performance degradation due to PDL can be largely mitigated without any coding overhead. Experimentally, this technique shows more than 2-dB $Q^{2}$ improvement for PDM-QPSK with 6-dB worst-case PDL.

OCIS codes: (060.1660) Coherent communications; (060.2330) Fiber optics communications.
\end{abstract}

\section{Introduction}

Polarization-division-multiplexed (PDM) coherent optical systems support 100-Gb/s and beyond, as powerful digital signal processing (DSP) techniques allow effective compensation of most of the system impairments [1]. Unfortunately, signal degradation due to polarization dependent loss (PDL) cannot be effectively compensated by DSP. After long-haul transmission, an accumulated PDL of several $\mathrm{dB}$ can occur, which may become the main system performance limiting factor. The study of PDL is generally limited to impairment analysis for system margin evaluation [2-4], and there is currently a lack of methods for improving performance in the presence of PDL. PDL causes non-orthogonality and ASE depolarization of the PDM signals [2], and while the non-orthogonality can be equalized by an adaptive polarization mode dispersion (PMD) equalizer, depolarized ASE noise degrades the OSNR of the lossy polarization, so its errors dominate the system performance. It has been suggested [3] that the data bits should be encoded together for both polarizations so that the total system performance is the average of both "good" and "bad" polarizations; however, the overall performance is still dominated by the lossy polarization, and so it is obviously desirable to somehow "reassign" ASE noise from the lossy polarization to the other polarization.

The degraded OSNR of the lossy polarization is similar to the scenario in wireless system where one channel suffers frequency selective fading; thus, PDL could be viewed as “polarization selective fading”. In MIMO wireless systems, pairing of sub-channels with different signal-to-interference-and-noise-ratios (SINR), using constellation rotation and real/imaginary interleaved pre-coding, improves the overall BER performance [5, 6]. This "pairwise coding” also improves the receiver sensitivity for direct-detection optical orthogonal-frequency-divisionmultiplexing [7]. Importantly, pairwise coding does not require an overhead, reducing payload data rate, and needs only a few extra computations per symbol, because only pairs of symbols are processed together.

In this paper, we propose and experimentally demonstrate that pairwise coding improves a PDM coherent optical system performance affected by PDL. By interleaving the real (I) and imaginary (Q) components of both polarizations, only the I or Q of each polarization suffers the "polarization selective fading", but the other component has a good OSNR. This evens out the performance of both polarizations. The experimental demonstrations are for single-channel and WDM PDM-QPSK systems, with a wide range of worst case PDL. We show that this novel polarization pairwise coding (PPC) scheme provides a large performance improvement over uncoded PDM-QPSK systems.

\section{Polarization pairwise coding and decoding}
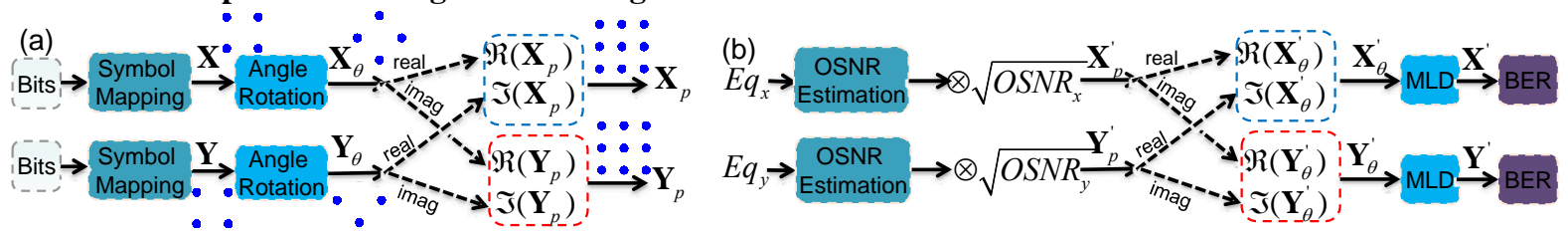

Fig. 1. (a) Transmitter polarization pairwise pre-coding, (b) receiver polarization pairwise decoding.

Figure 1(a) shows the proposed polarization pairwise pre-coding. Bit to symbol mapping is first performed for each polarization to produce the QAM streams $\mathbf{X}$ and $\mathbf{Y}$. The QAM symbols are then multiplied by a constant angle: $\mathbf{X}_{\theta}=\mathbf{X} \cdot e^{i \theta}, \mathbf{Y}_{\theta}=\mathbf{Y} \cdot e^{i \theta}$; the optimal rotation angle, $\theta_{o p t}$, is derived analytically in [6] for QPSK:

$$
\theta_{o p t}= \begin{cases}\pi / 4 & \lambda \leq 3 \\ \tan ^{-1}\left[(\lambda-1)-\sqrt{(\lambda-1)^{2}-\lambda}\right. & \lambda>3\end{cases}
$$


where $\lambda$ defines the receiver OSNR difference between two polarizations by $\lambda=\mathrm{OSNR}_{\text {good }} / \mathrm{OSNR}_{\text {bad }}$, and this rotation angle is designed to minimize the BER for a given OSNR difference between two polarizations. After angle rotation, I/Q component interleaving is used to generate the signals for each polarization before uploading to DAC: $\mathbf{X}_{p}=\mathfrak{R}\left(\mathbf{X}_{\theta}\right)+j \mathfrak{R}\left(\mathbf{Y}_{\theta}\right), \mathbf{Y}_{p}=\mathfrak{I}\left(\mathbf{X}_{\theta}\right)+j \mathfrak{I}\left(\mathbf{Y}_{\theta}\right)$, where $\mathfrak{R}(\bullet)$ and $\mathfrak{I}(\bullet)$ represent the real and imaginary parts of the signals, respectively. The six constellation diagrams in Fig.1a show the stages of polarization pairwise coding with PDM-QPSK and $45^{\circ}$ rotation angle. The transmitted symbol has a 9-QAM like constellation.

The receiver first implements standard DSP processes including: front-end correction, clock recovery, channel impairment compensation and carrier recovery. The equalized symbols $E q_{x} / E q_{y}$ are pairwise decoded, as shown in Fig. 1b. OSNR estimation is first performed for each polarization using the statistical moments method [8], and then the equalized signals are rescaled differently according to the OSNR of each polarization. Notably, in a real system, the OSNR difference varies due to the statistical nature of PDL, therefore the OSNR estimation and rescaling should be updated periodically, in accordance with the SOP rotation rate. A $\mathrm{kHz}$ update rate is reasonable for fiber with fast SOP rotation [9]. The I and Q components are then de-interleaved, and the resulting real and imaginary parts now suffer different noise levels. Maximum likelihood detection (MLD) is applied for symbol decision:

$$
\mathbf{X}^{\prime}=\arg \min _{\mathbf{C}_{k}}\left\{\left|\mathbf{X}_{\theta}^{\prime}-\mathbf{D}_{k}\right|^{2}\right\}, \quad \mathbf{Y}^{\prime}=\arg \min _{\mathbf{C}_{k}}\left\{\left|\mathbf{Y}_{\theta}^{\prime}-\mathbf{D}_{k}\right|^{2}\right\}, \quad \mathbf{D}_{k}=\mathfrak{R}\left(\mathbf{C}_{k} \cdot e^{i \theta}\right) \cdot \sqrt{S N R_{x}}+j \mathfrak{I}\left(\mathbf{C}_{k} \cdot e^{i \theta}\right) \cdot \sqrt{S N R_{y}}
$$

where: $\mathbf{C}_{k}$ is the constellation alphabet, i.e. $[1+j 1-j-1+1 j-1-1 j]$ for QPSK modulation, and $\mathbf{D}_{k}$ are the rotated and rescaled constellation points. The decisions are finally used for BER calculation.

\section{Experimental demonstrations}

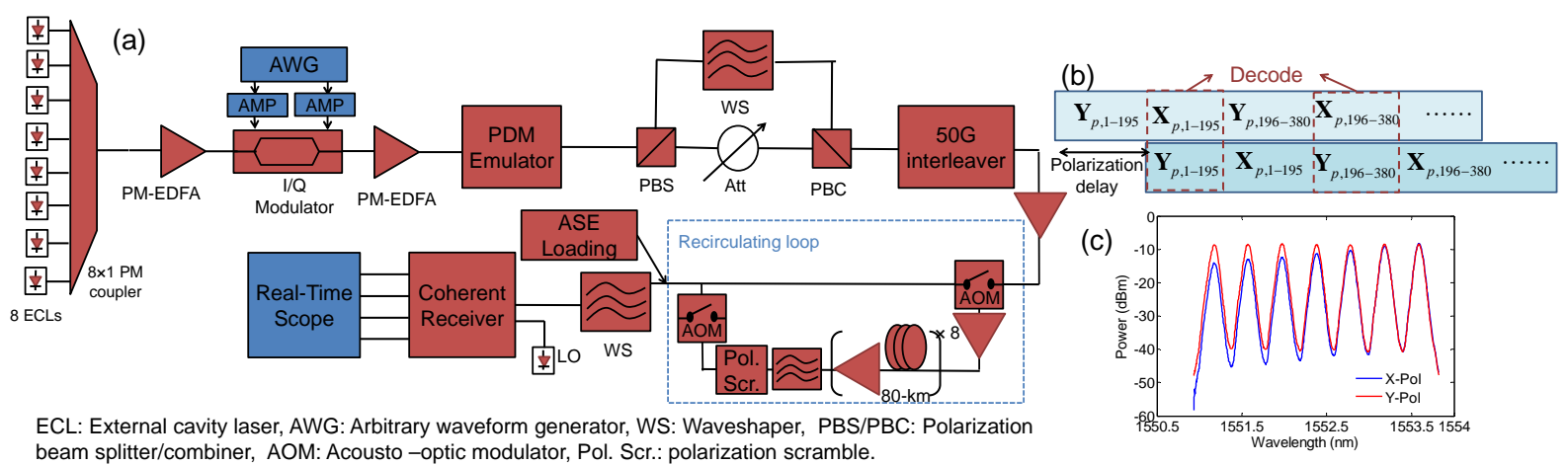

Fig. 2: (a) Experimental setup, (b) AWG input stream for PPC-QPSK, (c) X- and Y-polarization spectra of WDM PPC-QPSK.

Figure 2a shows the setup for 10-Gbaud single channel and 8-channel WDM PDM-QPSK experiments. At the transmitter, eight ECLs with $50-\mathrm{GHz}$ carrier spacing were multiplexed with an $8 \times 1$ polarization-maintaining (PM) coupler. The baseband signals were generated by a 10-GSa/s arbitrary waveform generator with either conventional PDM-QPSK or the proposed PPC-QPSK. Since we could only program our signals in one polarization due to equipment limitations, for PPC-QPSK, $\mathbf{X}_{p}$ and $\mathbf{Y}_{p}$ were blocked with every 195 symbols and interleaved together for uploading to the AWG as shown in Fig. 2b. The 195-symbol offsets match the delay between two polarizations, therefore the PDM emulator outputs have matched PPC blocks every 195 symbols. A PBS was used to split the signals into two polarizations, then a Finisar WaveShaper (WS) was used to generate single-channel or multichannel PDL in the X-polarization. Frequency dependent multi-channel PDL generated (7 to $0 \mathrm{~dB}$ PDL on Channels 1-8); Fig. 2c shows the X- and Y-polarization spectra of the WDM signal. An optical attenuator was used in Ypolarization to match the insertion loss of this WS. Finally, a PBC recombines the two polarizations. All the connectors and optical devices between the lasers and the PDL element were polarization maintaining, so the emulated PDL refers to the lumped worst case PDL $\left(0^{\circ}\right.$ between signal polarizations and the PDL lossy axis) [2]. Then, after a 50-GHz channel interleaver, the optical signal was transmitted through a recirculating loop, consisting of two acousto-optic modulator (AOM) switches, 640-km SSMF, EDFAs and a gain-flattening WS. ASE noise was inserted for the single-channel back-to-back (B2B) case, and another WS was used to select the desired channel for coherent detection. Finally, a real-time oscilloscope was used to digitize the signals. The receiver DSP used the same modulation format transparent training-aided methods [10] for both PDM-QPSK and PPC-QPSK schemes. Note that only the matched signal blocks of the equalized PPC-QPSK signals were selected for PPC decoding and BER calculations. 

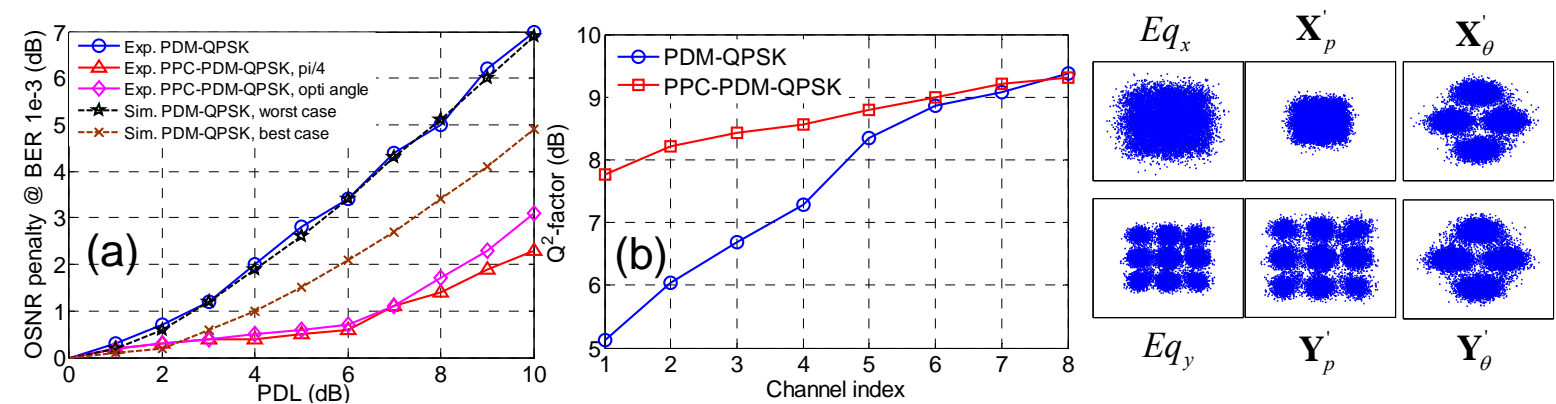

Fig. 3. Experimental results for: (a) OSNR penalty for single-channel B2B transmission; (b) WDM transmission after 5120-km for PDM-QPSK and PPC-QPSK signals. The constellation diagrams are showing the PPC decoding process at B2B with 11.1-dB OSNR and 6-dB PDL.

Figure 3a shows the measured OSNR penalty $(0.1 \mathrm{~nm})$ for a BER of $10^{-3}$ (the average BER of two polarizations) versus different PDL values for single-channel B2B. The solid and dashed curves show experimental and simulation results, respectively. The PDM-QPSK experimental results closely match the simulation results with worst case PDL, where a 3.5-dB OSNR penalty occurs with PDL value of $7 \mathrm{~dB}$. The proposed PPC-QPSK using $45^{\circ}$ rotation angle outperforms the PDM-QPSK, showing just over 1-dB OSNR penalty with 7-dB PDL. Moreover, the $45^{\circ}$ rotation angle shows almost the same performance as the optimal angle, when the PDL is less than 7-dB, and so can be used for all cases to avoid the need for a feedback path from receiver to transmitter. As a reference, the simulated PDM-QPSK BERs with best case PDL ( $45^{\circ}$ between signal polarization and the lossy axis) are also plotted; the proposed PPC technique is still able to provide 0.3/1.6/2.3-dB OSNR advantage at 3/6/9-dB PDLs.

Figure 3b shows the measured $Q^{2}$-factors $\left(Q^{2}(d B)=20 \log _{10}\left(\sqrt{2} e r f c^{-1}(2 B E R)\right)\right)$ for WDM transmission, where the PPC-QPSK shows more than 2.5-dB $Q^{2}$ improvement for Channel 1 (7-dB PDL). The six constellation diagrams of Fig. 3 show the PPC decoding process at B2B with 11.1-dB OSNR and 6-dB PDL: (left column) equalized signals, (middle) signals after rescaling and (right) after de-interleaving. It is worth emphasizing that although the experiment only contains worst case lumped PDL, which causes a fixed OSNR difference, while the practical distributed PDL with random angle between signal polarizations and PDL axes leads to time-varying OSNR difference; however, the proposed method can still be effective since the I/Q de-interleaver always effectively distributes the ASE between the two polarizations of a given channel, and then the PPC decoding enables adaptive PDL mitigation after accurately estimating the OSNR.

\section{Conclusions}

We propose polarization pairwise coding for PDM coherent optical systems, to improve the transmission performance in the presence of PDL. By rotating the original information symbols and interleave the real and imaginary components between two polarizations of a given channel, the decoded signals from both polarizations can have similarly low error rates, which is always better than summing the widely-varying error rates of good and bad signals. Both the single-channel and the WDM PDM-QPSK experimental results show that the proposed method greatly enhances the overall system performance under a wide range of PDL, without any coding overhead.

\section{Acknowledgements}

This work is supported by the Australian Research Council’s Laureate Fellowship Grant (FL13010041).

\section{References}

[1] S. J. Savory, "Digital coherent optical receivers: algorithms and subsystems,” IEEE J. Sel. Top. Quantum Electron. 16(5), 1164-1179 (2010).

[2] C. Xie, "Polarization-dependent loss induced penalties in PDM-QPSK coherent optical communication systems,” in Proc. Opt. Fiber Comm. Conf. (OFC), paper OWE6 (2010).

[3] T. Duthel, et al., "Impact of polarization dependent loss on coherent POLMUX-NRZ-QPSK," in Proc. Opt. Fiber Commun. Conf. (OFC), paper OThU5 (2008).

[4] C. Xie, "Impact of nonlinear and polarization effects in coherent systems,” Opt. Express 19(26), B915-B930 (2011)

[5] S. K. Mohammed, et al., "MIMO precoding with X- and Y-codes,” IEEE Trans. Inf. Theory 57(6), 3542-3566 (2011).

[6] J. Boutros, et al., "Signal space diversity: a power- and bandwidth-efficient diversity technique for the Rayleigh fading channel," IEEE Trans. Inf. Theory 44(4), 1453-1467 (1998).

[7] Y. Hong, et al., "Sensitivity improvement and carrier power reduction in direct-detection optical OFDM systems by subcarrier pairing," Opt. Express 20(2), 1635-1648 (2012).

[8] C. Zhu, et al., "Statistical moments based OSNR monitoring for coherent optical systems,” Opt. Express 20(16), 17711-17721 (2012).

[9] P. M. Krummrich, et al., "Field trial results on statistics of fast polarization changes in long haul WDM transmission systems,” in Proc. Opt. Fiber Comm. Conf. (OFC), paper OThT6 (2004).

[10] C. Zhu, et al., "Low-complexity fractionally-spaced frequency domain equalization with improved channel estimation for long-haul coherent optical systems,” in Proc. Opt. Fiber Comm. Conf. (OFC), paper OW4B.5 (2012). 